\title{
Von der chinesischen Konzeption "Ein Land, zwei Systeme"
}

\author{
Von Rongyuan Lin
}

\section{Einleitung}

Die Gemeinsame Erklärung der Regierung der Volksrepublik China und der Regierung des Vereinigten Königreichs von Großbritannien und Nordirland über die Hongkong-Frage 1 und die Gemeinsame Erklärung der Regierung der Volksrepublik China und der Regierung der Republica Portuguesa über die Macao-Frage 2 werden sowohl von der chinesischen wie auch von der britischen bzw. von der portugiesischen Seite hoch bewertet. Diese beiden Vereinbarungen konnten im Grunde genommen erst auf der Basis der chinesischen Konzeption "ein Land, zwei Systeme" erreicht werden. 3

Das Hongkong-Problem ist für beide Seiten eine harte Nuß gewesen. Die VR China ist ein sozialistischer Staat, und sie wollte bis 1997 die Souveränität über Hongkong wiedererlangen. Andererseits ist das unter der britischen Herrschaft stehende Hongkong gegenwärtig eine kapitalistische Gesellschaft. Wie kann man beides unter ein Dach bringen, ohne dem einen oder dem anderen zu schaden? Unter diesen Umständen ist auf die historischen Hintergründe, die gegenwärtige Realität in Hongkong und die Interessen aller Seiten Rücksicht zu nehmen. Die hauptsächlich von Deng Xiaoping konzipierte Formel "ein Land, zwei Systeme"4 stellt somit die Grundlinie dar, die von allen Seiten leichter angenommen werden könnte. Nach chinesischer Ansicht sollte mit diesem Konzept nicht nur die Rückgabe der Souveränität über Hongkong an China ermöglicht werden, sondern es könnte auch eine feste Grundlage für die Stabilität und langfristige Prosperität in Hongkong bilden. ${ }^{5}$ Mit der Lösung der Hongkong-Frage war es leichter, die Macao-Frage zu regeln. Das Vorbild von Hongkong sollte ebenso ein Beispiel für die Lösung des Taiwan-Problems sein.6 Úber die Konzeption "ein Land, zwei Systeme" wird seit Jahren in China und in Hongkong

1 Die deutsche Übersetzung des Textes in: Beijing Rundschau (weiterhin als BR), Nr. 41, 09.10.1984.

2 Die deutsche Übersetzung des Textes in: BR, Nr. 14, 07.04.1987.

3 Yan Mingfu, Die erste Phase des Sozialismus und die Einheitsfront, in: Yiu Shi (Praxis), Nr. 2, 1988, S. 9 f.

4 Deng Xiaoping, Über die Konzeption "ein Land, zwei Systeme", in: Wenhuibao (Hongkong), 31.01.1987.

5 Vgl. BR, Nr. 42, 16.10.1984, S. 6.

6 Vgl. Helmut Schmidt, Einleitung zu Deng Xiaopings "Die Revolution der Reform", Berlin 1988, S. 13. 
lebhaft diskutiert. ${ }^{7}$ Denn mit diesem Konzept stehen wir einem ganz neuen Phänomen gegenüber. In der vorliegenden Arbeit wird versucht, eine allgemeine Darstellung und Analyse der Konzeption "ein Land, zwei Systeme" zu geben.

\section{Vorgeschichte der Konzeption}

Die VR China wurde am 1.10.1949 gegründet. Vor der Gründung des Neuen Chinas nahmen die Guomindang-Behörden mit Jiang Jieshi (Chang Kaishek) an der Spitze Taiwan und einige kleine Inseln als ihre letzte Zuflucht. Mit der Unterstützung der USA, vor allem militärisch, konnten und können sie ihre Macht auf Taiwan aufrechterhalten. Mit Gewalt Taiwan zu befreien, war in den 50er und 60er Jahren wegen der militärischen Präsenz der USA auf Taiwan, vor allem der 7. Kriegsflotte in der Taiwan-Straße, für die junge Volksrepublik auch kaum möglich. Auf diese Weise ist China seit 1949 gespalten. 8 Die chinesische Regierung hat in den 40 Jahren jedoch nie auf die Wiedervereinigung verzichtet 9 - und die Behörden in Taiwan taten das genauso, sie bestehen wenigstens verbal auf einer Vereinigung unter den angeblichen "Drei Volksprinzipien"10. Die diesbezüglichen Richtlinien der chinesischen Regierung hat verschiedene Phasen erfahren.

Kurz nach der Staatsgründung lag der Akzent der Richtlinie auf der "Befreiung Taiwans"11, wobei diese hauptsächlich mit Gewalt erreicht werden sollte. Die Losung lautete damals: "Wir werden unbedingt Taiwan befreien!" Erst im Mai 1955 sprach der damalige Ministerpräsident Zhou Enlai von zwei Möglichkeiten hinsichtlich der Regelung des Taiwan-Problems, nämlich der auf friedlichem Weg und der mit Waffengewalt.12 Diese Zwei-Möglichkeiten-Formel bleibt seitdem nicht verändert, wobei man die friedliche Lösung seit Anfang der 70er Jahre stärkstens betont, ohne allerdings eine feste Zusage zur friedlichen Regelung der Taiwan-Frage zu geben. Denn nach Auffassung von Deng Xiaoping hoffe Beijing auf eine Lösung mit Friedensmitteln, aber mit einer derartigen festen Zusage würde China sich zu sehr die Hände binden.13 Trotzdem ist eine gewaltige Wendung in der Taiwan-Politik Beijings in den letzten 10 Jahren deutlich bemerkbar. Sie

7 Vgl. Liaowang-Zeitschrift (Wachposten), 07.04., 16.11., 23.11., 30.11.1987, und ebenso The Nineties (Hongkong), Nr. 5, 1985, S. 52 ff., Nr. 12, 1985, S. 31 ff., Nr. 1, 1988, S. 66 ff.

8 Vgl. Rongyuan Lin, Die Beziehungen zwischen China und Deutschland, Baden-Baden 1986, S. $98 \mathrm{ff}$.

9 Deng Xiaoping, Aufbau des Sozialismus mit chinesischen Eigenschaften, 2. erweiterte Auflage, Beijing 1987, S. 48.

10 Zili Zaobao (Selbständige Morgenzeitung, Taiwan), 12.04.1989.

11 ZhouTuo und Wei Daye, Abriß über die wichtigen Ereignisse in Taiwan, Beijing 1982, S. 60.

12 Ebenda, S. 64.

13 BR, Nr. 2, 16.01.1979, S. 17. 
erfolgte parallel zu der gesamten Reform- und Öfnungspolitik nach außen, die auf der 3 . Tagung des XI. ZK der KP Chinas 1978 formuliert wurde.14

Die Aufnahme diplomatischer Beziehungen zwischen der VR China und den USA am 1.1.1979 hat sicherlich zu der Wendung der Taiwan-Politik der chinesischen Führung in nicht unbedeutendem Maße beigetragen. In dem Kommunique über die Herstellung diplomatischer Beziehungen zwischen Beijing und Washington erkennt die US-Regierung "die Regierung der VR China als die einzig legitime Regierung Chinas" an. In diesem Rahmen unterhalte die Bevölkerung der USA mit der Bevölkerung Taiwans kulturelle, Handels- und andere inoffizielle Beziehungen ... "Die Regierung der Vereinigten Staaten von Amerika erkennt den Standpunkt an, daß es nur ein China gibt und daß Taiwan ein Teil Chinas ist." 15

In seiner "Botschaft an die Landsleute auf Taiwan" vom 1. Januar 1979 erklärte der Ständige Ausschuß des Volkskongresses der VR China u.a.: Bei der Lösung der TaiwanFrage würden die chinesischen Führer "den gegenwärtigen Zustand und die Meinungen aller Bevölkerungsschichten auf Taiwan respektieren und sich um eine vernünftige Politik und Methode bemühen, damit die Bevölkerung Taiwans keinen Schaden nehmen" könnte. 16

Kurz vor und nach der Aufnahme diplomatischer Beziehungen mit den USA zog Deng Xiaoping bereits die Notwendigkeit einer dritten Zusammenarbeit mit der Guomindang in Betracht. Er versicherte u.a., nach der Wiedervereinigung würden die Gesellschaftsordnung, das wirtschaftliche System, die Lebensweise und ausländische Investitionen in Taiwan unangetastet bleiben, was in der Tat zum ersten Mal den Urgedanken über die Formel "ein Land, zwei Systeme" zum Ausdruck brachte. 17

Es war wiederum Deng Xiaoping, der a m 30.1.1979 in den USA verkündete: "Wir gebrauchen die Wendung 'Befreiung Taiwans' nicht mehr. Wenn Taiwan ins Vaterland zurückkehrt, werden wir die gegenwärtige Realität und das bestehende System dort respektieren."18 Von da an ist die Losung "Wir werden unbedingt Taiwan befreien!" aus der chinesischen Presse und dem politischen Leben Chinas völlig verschwunden.

14 Huan Xiang, Ein Abkommen von historischer Bedeutung, in: BR, Nr. 14, 09.10.1984, S. 20 f., hier S. 20.

15 Renmin Ribao (Die Volkszeitung, weiterhin als RMRB), 17.12.1978, und ebenso BR, Nr. 51, 26.12.1978.

16 RMRB, 01.01.1979.

17 Yan Jiaqi, Die Konzeption "ein Land, zwei Systeme" und der Weg zur Vereinigung Chinas, in: Die Macht und die Wahrheit, Beijing 1987, S. 183 ff., hier S. 1984.

18 BR, Nr. 6, 13.02.1979, S. 12. 
Konkretisiert wurde die friedliche Lösung des Taiwan-Problems jedoch erst zwei Jahre später, als Ye Jiangying, der damalige Vorsitzende des Ständigen Ausschusses des Volkskongresses, in einem Interview mit der Xinhua-Nachrichtenagentur den 9-PunkteVorschlag bekanntgab. Darin war eine konkrete Idee über "ein Land mit zwei Systemen" deutlich sichtbar. Der dritte Punkt versicherte, nach der Wiedervereinigung könnte Taiwan "als Region unter besonderer Verwaltung eine weitestgehende Autonomie genießen und seine Streitkräfte beibehalten. Die Zentrale Regierung wird sich nicht in die regionalen Angelegenheiten einmischen." Im Punkt 4 wurde wiederholt erklärt: "Das gegenwärtige Gesellschafts- und Wirtschaftssystem Taiwans, seine Lebensweise und seine wirtschaftlichen und kulturellen Beziehungen mit dem Ausland bleiben unverändert. Die Eigentumsrechte und das legitime Erbrecht auf Privateigentum, Häuser, Grund und Boden und auf Betriebe sowie ausländische Kapitalanlagen bleiben unangetastet."19

Deng Xiaoping faßte diesen Vorschlag als Konzeption "ein Land, zwei Systeme" zusammen - das war das erste Mal, daß die Formulierung "ein Land, zwei Systeme" of fiziell bekannt wurde. Er sagte u.a., zwei Systeme seien zulässig, und dies gelte nicht nur für die Taiwan-Frage, sondern auch für das Hongkong-Problem. 20

Um die Konzeption "ein Land, zwei Systeme" zu rechtfertigen und ihre Anwendung zu ermöglichen, kam es 1982 zu einer Verfassungsreform in der VR China. In Art. 31 der neuen Verfassung heißt es: "Der Staat kann, wenn nötig, Sonderverwaltungsgebiete einrichten. Die in den Sonderverwaltungsgebieten einzurichtenden Systeme sollen den gegebenen Verhältnissen entsprechend vom Nationalen Volkskongreß gesetzlich festgelegt werden." 21 Auf diese Weise wurde die rechtliche Basis für die Konzeption "ein Land, zwei Systeme" geschaffen.

Aus der oben dargestellten Chronik geht hervor, daß die Konzeption "ein Land, zwei Systeme" ursprünglich vor allem für die Lösung des Taiwan-Problems entwickelt worden ist.22 Sie hat aber zuerst in der Hongkong- bzw. in der Macao-Frage ihre Anwendung gefunden, weil die Behörden auf Taiwan sie immer noch ablehnen und weil die Hongkongund die Macao-Frage unter dem Zeitdruck zuerst in den Beziehungen zwischen der VR China und Großbritannien sowie zwischen der VR China und Portugal auf die Tagesordnung gelangten. Somit hat die Formel "ein Land, zwei Systeme" die Gestalt einer konkreten politischen Linie angenommen. Sie ist zu einer Politik, ja einer Grundpolitik, geworden,

19 RMRB, 31.09.1981.

20 s. Anm. 9, S. 91.

21 Die deutsche Übersetzung der Verfassung der VR China 1982 in: BR, Nr. 52, 28.12.1982, hier S. 13.

22 s. Anm. 9, S. 91. 
wie sie in der Gemeinsamen Erklärung zwischen Beijing und Lissabon Ausdruck gefunden hat. 23

\section{Definition aus chinesischer Sicht}

Über die Formel "ein Land, zwei Systeme" wird seit ihrer Bekanntmachung lebhaft diskutiert. Die Auseinandersetzung konzentriert sich vor allem auf die Fragen: Wie wird die Konzeption "ein Land, zwei Systeme" definiert? Kennt die Geschichte bereits derartige Erscheinungen? Ist sie unter den gegenwärtigen Verhältnissen Chinas durchführbar? Die Auffassungen sind ganz unterschiedlich.24

Was die Definition des chinesischen Originals "Yi Guo Liang Zhi" betrifft, stößt man schon sprachlich auf Schwierigkeiten. Denn unter dem chinesischen Schriftzeichen "Guo" können in diesem Fall drei Begriffe verstanden werden: das Land, die Nation und der Staat. Hier scheint für die meisten chinesischen Autoren der territoriale bzw. geographische Begriff "Land" vorgezogen zu werden, wenn sie die Formel "ein Land, zwei Systeme" als eine "in der Geschichte nie dagewesene Schöpfung" bewerten. $25 \mathrm{Da}$ in einer Nation zwei oder mehrere Staaten nebeneinander bestehen, kennt nicht nur die Geschichte, sondern auch die Gegenwart, z.B. die arabische Nation umfaßt mehrere Staaten und verschiedene Gesellschaftstypen. Aber der Begriff "Staat" wird hier nicht völlig ausgeschlossen, obwohl er mit zwei politischen Systemen schwer vereinbar wäre, weil die chinesische Regierung seit jeher gegen die "zwei-China"-Theorie ist und Taiwan nie als "Staat" anerkennt.26 Wenn man also das "Guo" als "Land" auffaßt, würde es heißen: Auf dem chinesischen Territorium werden zwei unterschiedliche Gesellschafts-, Wirtschafts- und politische Systeme nebenund miteinander bestehen. Wird unter dem "Guo" der Begriff "Staat" verstanden würde es in diesem Zusammenhang heißen: Unter einer zentralen Staatsgewalt werden zwei unterschiedliche Systeme friedlich und miteinander existieren.

Kennt die Geschichte die Erscheinung "ein Land, zwei Systeme"? Die einen sagen: ja, und nehmen ein Beispiel aus der chinesischen Geschichte, und zwar aus der Liao-Dynastie (907-1125). Der Kaiser Liao Taizong (Amtszeit: 938-947) praktizierte zwei politische Systeme: das eigentlich bestehende Staatssystem zur Herrschaft über die Qidan-Nationalität und das Han-System zur Herrschaft über die Han-Nationalität in den neu besetzten Gebie-

23 s. Anm. 2.

24 Vgl. Wang Bangzuo und Wang Huling, Von dem Verhältnis zwischen der Souveränität und der Regierungsgewalt bei der Konzeption "ein Land, zwei Systeme", in: Studien der Politischen Wissenschaft, Nr. 2, 1985, S. 12 ff., hier S. 12; vgl. ebenso Weng Songran, Von der Formel "ein Land, zwei Systeme", in: The Nineties, Nr. 12, 1985, S. $30 \mathrm{ff}$.

$25 \mathrm{Vgl}$. Wang Bangzuo und Wang Huling, s. Anm. 24.

26 Vgl. Kommuniqué zwischen der VR China und den USA in Shanghai, in: RMRB, 28.02.1972. 
ten Yanzhou, Yingzhou u.a. Außerdem hätte es vor dem Krieg 1861-1865 in den USA auch zwei Gesellschaftsordnungen gegeben. Manche sind sogar der Meinung, daß auf dem chinesischen Festland gegenwärtig ebenso zwei verschiedene Wirtschaftstypen vorhanden seien, nämlich das sozialistische Gemeineigentum und das Privateigentum, und daß dies genauso "ein Land, zwei Systeme" bedeuten würde. 27

Die meisten Autoren auf dem Festland Chinas sind jedoch der anderen Auffassung. Sie behaupten, daß Konzept "ein Land, zwei Systeme" sei ein "ganz neuer Begriff". Es sei eine "phantasievolle und geniale Schöpfung", die in der Geschichte der Politwissenschaft keine Parallele kenne.28

Gegenüber den großen Meinungsverschiedenheiten versuchte der Direktor des Instituts für Politische Wissenschaft der allchinesischen Akademie für Gesellschaftswissenschaften, Yan Jiaqi, von der Politwissenschaft ausgehend die Formel "ein Land, zwei Systeme" zu definieren oder ihr eine "wissenschaftliche Zusammenfassung" zu geben, wie er es nannte. 29

Seiner Auffassung nach sollte die Konzeption "ein Land, zwei Systeme" wie folgend definiert werden: In einem Land würden gemäß der Bestimmungen und Gesetze unterschiedliche politische, wirtschaftliche und Gesellschaftssysteme in verschiedenen Landesteilen praktiziert werden. Aber die Regierungen in diesen Gebieten sollten ausschließlich die örtlichen administrativen Verwaltungen oder regionale Regierungen sein, die nicht die Staatssouveränität ausüben dürften. 30 Deshalb sollte die Formel unter vier Aspekten konkretisiert werden:

1. Der Satz "ein Land, zwei Systeme" müßte verfassungs- und gesetzmäßig unmißverständlich festgelegt werden. Die verschiedenen Systeme sollten relativ langfristig und stabil sein. Sollten Streitigkeiten zwischen den verschiedenen Gebieten auftreten, müßten sie beide Seiten den Gesetzen gemäß mit friedlichen Mitteln beilegen. 31

2. Mit der Formel "ein Land, zwei Systeme" würde gemeint werden, daß unterschiedliche Systeme in verschiedenen Gebieten eingeführt werden sollten. In Großbritannien bestünden die Erbmonarchie und die parlamentarische Demokratie parallel. Auf dem chinesischen Festland seien in der Wirtschaftsordnung mit dem sozialistischen Gemein-

27 s. Anm. 17.

28 s. Anm. 25.

29 s. Anm. 17.

30 Ebenda; vgl. auch Chen Hangcheng, Die Konzeption "ein Land, zwei Systeme" ist eine wichtige Entwicklung des Sozialismus, in: Joumal der Universität Zhengzhou (philosophische und gesellschaftswissenschaftliche Ausgabe), Nr. 6, 1988, S. 6 ff., hier S. 7.

31 Ebenda. 
eigentumssystem als Basis verschiedene Wirtschafts- und Eigentumsformen vorhanden. Diese beiden Fälle könnten nicht als Beispiel "ein Land, zwei Systeme" bewertet werden. 32

3. Mit der Formel sollte gemeint sein, daß die politischen, die wirtschaftlichen und die Gesellschaftssysteme, die in verschiedenen Gebieten praktiziert würden, deutlich und wesentlich unterschiedlich sein müßten. In Großbritannien existierten verschiedene Rechtssysteme - das allgemeine Rechtssystem in England und das kontinentale Rechtssystem in Schottland -, dies wäre nicht mit der Formel "ein Land, zwei Systeme" gleichzusetzen. In den chinesischen Sonderwirtschaftszonen an der Küste sei nur das wirtschaftliche System von dem im Inland wesentlich unterschiedlich. Dies bedeute noch nicht, das Konzept "ein Land, zwei Systeme" einzuführen. 33

4. Die Gebiete, in denen unterschiedliche Systeme praktiziert würden, seien Bestandteile des einheitlichen Staates, deren Verwaltungen nicht gestattet würde, die Souveränität des Staates auszuüben, z.B. die Befugnisse hinsichtlich der Außenpolitik, der Landesverteidigung, der Kriegserklärung und des Friedenschließens. 34

Von dieser "wissenschaftlichen Definition" aus seien die obengenannten Beispiele aus der chinesischen Liao-Dynastie und der amerikanischen Geschichte kein Maßstab. Denn in den beiden Fällen hätte es in dem Verhältnis zwischen verschiedenen Gebieten an verfassungsmäßigen und gesetzlichen Bestimmungen gemangelt, die diesen eine langfristige Stabilität hätten garantieren können. Ihre Beziehungen zueinander seien instabil und gespannt gewesen, und sie hätten oft zu Kriegen geführt. Die beiden Beispiele könnten höchsten als "Nebeneinanderbestehen zweier Systeme" betrachtet werden. 35

Wie oben erwähnt, wurde die Konzeption "ein Land, zwei Systeme" mit dem Ziel entwickelt, die Taiwan-, die Hongkong- und die Macao-Frage auf friedlichem Weg zu regeln. Sollte sie praktiziert werden, sollte sie sich nach chinesischer Auffassung durch folgendes auszeichnen:

1. Sie sollte unter ein und derselben Staatsstruktur praktiziert werden, die gewisse föderalistische Eigenschaften besitzen sollte.

2. Bei ihrer Einführung sollte der Sozialismus das Übergewicht haben. Es sollte heißen: Auf dem Festland sollte sich der Sozialismus weiterhin entwickeln, während in 
Hongkong, Taiwan usw. nach deren Vereinigung mit dem Festland der Kapitalismus nach wie vor beibehalten werden könnte.36

Ein Hongkonger Politologe versuchte auf dieser Basis die Definition exakter zu formulieren. Er sagte u.a.: "Die Definition von 'ein Land, zwei Systeme' sollte heißen: In einem einheitlichen Staat wird einem Landesteil oder mehreren Landesteilen gestattet, den Bestimmungen der Verfassung und Gesetzen gemäß politische, wirtschaftliche und gesellschaftliche Systeme einzuführen, die wesentlich unterschiedlich von dem allgemeinen System des Staates sind. Die Regierungen in diesen Gebieten sind jedoch regionale Regierungen und sie dürfen nicht die Staatssouveränität ausüben, es sei denn, die Zentralgewalt ermächtigt sie, dies zu tun." 37

Zudem hat er auch auf die Exklusivität der chinesischen Definition von "ein Land, zwei Systeme" hingewiesen. Sie schließe nicht nur alle Vereinigungsmodelle aus, die auf dem "nicht-ein-China"-Prinzip beruhen, sondem auch andere Modelle und Vorschläge, die seiner Auffassung nach relativ milde und vernünftig seien, wie das "Bundesstaat-Modell", das "Staatenbund-Modell", das Modell "eine Souveränität, zwei Regierungsgewalten" sowie das Modell "ein Land mit mehreren Systemen". Alles, was dem unitarischen Prinzip und dem Grundsatz, daß ausschließlich die Zentralregierung die Souveränität ausüben darf, zuwiderläuft, könnte die chinesische Führung bei der Interpretation ihrer Konzeption "ein Land, zwei Systeme" nicht hinnehmen und dulden. 38

\section{Verschiedene Modelle zur Wiedervereinigung}

Bei der Auseinandersetzung über "ein Land, zwei Systeme" betonen die Politiker und Wissenschaftler auf dem Festland, daß diese Konzeption durchführbar sei39, während ihre Kollegen in Hongkong, Taiwan und im Ausland große Schwierigkeiten auf dem Weg der Verwirklichung dieser Konzeption sehen, wenn sie sie nicht überhaupt für unmöglich halten. Dies gilt besonders für Taiwan. Namentlich hier werden die wesentlichen Unterschiede der politischen, wirtschaftlichen und gesellschaftlichen Systeme hervorgehoben, die sich seit 40 Jahren auf beiden Seiten der Taiwan-Straße entwickelt haben; deshalb hält man es für notwendig, ein Ubergangssystem einzurichten.40 Auf diese Weise sind verschiedene Modelle und Konzepte für die Formel "ein Land, zwei Systeme" in der

36 Ebenda.

37 Weng Songran, s. Anm. 24.

38 Ebenda.

39 s. Anm. 9, 17 und 25.

40 Vgl. Shen Junshan, Eine Wohnung, zwei Zimmer, jeder hat seine Schlüssel, in: The Nineties, Nr. 5, 1985, S. 41 ff., Weng Songran, s. Anm. 24, sowie Qiu Chuiling, Interview mit Yan Jiaqi über politische Systeme und politische Reformen, in: The Nineties, Nr. 12, 1986, S. 40 ff. 
Diskussion, z.B. das "Deutschland-Modell", das "Singapur-Modell", das "FinnlandModell", das "Olympiade-Modell", das "Bundesstaat-Modell", das "Staatenbund-Modell", "Vereinigung durch Demokratie", "Vereinigung durch Entwicklung der Wirtschaft", das Konzept "ein Land mit mehreren Systemen bei einer doppelten Anerkennung", das "Europäische Gemeinschaft-Modell", die Formel "ein Land mit zwei ebenbürtigen Regierungen" usw., um nur einige Beispiele zu nennen.41

Auf all diese Modelle einzugehen ist hier schwierig und auch nicht nötig. Aber einige Beispiele zu schildern und zu analysieren scheint zweckmäßig, um die Formel "ein Land, zwei Systeme" besser zu verstehen.

Das "Deutschland-Modell" ist von Professor Xiao Xinyi aus Kanada aufgestellt worden42, wonach sich das chinesische Festland und Taiwan wie die beiden Staaten in Deutschland einander als Staat oder Staatlichkeit anerkennen sollten. In den gespaltenen Ländern der heutigen Welt sei die Art und Weise, in der die Deutschen ihre Probleme behandeln, die vernünftigste. Für alle Chinesen lohne es sich am besten, sie gewissenhaft zu studieren und zu forschen. 43 Die chinesische Führung könnte nun jedoch das "Deutschland-Modell" nicht annehmen, während die Behörden auf Taiwan das Konzept "ein Land, zwei Systeme" genauso ablehnen wollten. Es sei notwendig, einen Mittelweg zu finden: in der 1. Phase das "Deutschland-Modell" einzuführen und dann in der 2. Phase die Konzeption "ein Land, zwei Systeme" zu verwirklichen.44

Das "Olympiade-Modell" wird auch viel diskutiert, wonach das chinesische Festland und Taiwan gleichzeitig intemationalen Organisationen beitreten könnten, wie sie seit 1981 gleichzeitig an internationalen Sportveranstaltungen teilnehmen, wobei das Festland den Namen "die VR China" führt, während Taiwan den Namen "Chinese Taibei" trägt.

Das "Olympiade-Modell" ist von Professor Shen Junshan vielseitig dargestellt und entwickelt. Die Formel "ein Land, zwei Mannschaften" sollte sich zu der Formel "ein Land, zwei Sitze" weiterentwickeln. 45 Nachdem die VR China 1986 als einzig legitimer Vertreter Chinas in die Asien Development Bank eingetreten ist, kann Taiwan unter dem Namen "Chinese Taibei" weiterhin in dieser Organisation bleiben.46 Die Behörden auf Taiwan

41 Ebenda; s. auch Anm. 17 und 25. Vgl. ebenso Xinbao (Hongkong), 11.04.1989, Zili Zaobao, 12.04.1989 und Zili Wanbao (Selbständige Abendzeitung, Taiwan), 17.04.1989.

42 Vgl. Qiu Chuiliang, s. Anm. 40, S. 46.

43 s. Anm. 17 und 25.

44 s. Anm. 42.

45 s. Anm. 37 und 40.

46 RMRB, 06.05.1989. Vgl. ebenso Yang Liyu, Von der Konzeption "ein Land, zwei Mannschaften" zu der Formel "ein Land, zwei Sitze", in: The Nineties, Nr. 1, 1985, un China aktuell, Nr. 5, 1985, S. 285 f., s. auch Shen Junshan, Anm. 40. 
scheinen diese Formel leichter hinzunehmen, um international eine noch schlimmere Isolierung $\mathrm{zu}$ vermeiden, während Beijing aufgrund der "Einheitsfront"-Politik die Taktik geändert habe und nicht mehr darauf bestehe, daß Taiwan aus einer internationalen Organisation austreten müsse, wenn Beijing ihr beitreten wollte. 47

Nach der Auffassung von Shen Junshan sollte eine Lage "ein Land, drei Systeme" geschaffen werden, wenn sich Taiwan mit dem Festland vereinigt haben würde. Wirtschaftlich könnte von der Formel "ein Land, zwei Systeme" die Rede sein. Denn sowohl in Hongkong als auch in Taiwan bestehe die kapitalistische Wirtschaft, während sich die sozialistische Wirtschaft auf dem chinesischen Festland entwickele. Dies gelte jedoch keineswegs für die politischen Systeme. Hongkong würde nach dem Abzug der Briten keine andere Wahl übrigbleiben, als sich mit dem Festland zu vereinigen und sich mit dem Status eines "Sonderverwaltungsgebietes" abzufinden. Taiwan wolle sich sicher nicht in einen Status einer regionalen Regierung emiedrigen lassen. Das politische System in Taiwan müsse nach der eventuellen Vereinigung anders sein als das in Hongkong. Seiner Vision nach lehne Taiwan das Konzept "ein Land, zwei Systeme" ab. Es könnte aber die Formel "one China, two entities" annehmen. In diesem Fall würden die "two entities", "separate but not independent" sein. Die "two entities" würden "gemeinsam die Staatssouveränität ausüben" und "jeder Teil könnte die Regierungsgewalt nur in seinem Teilgebiet für sich allein in Anspruch nehmen".48

Shen Junshan hat eine anschauliche Schilderung von der Formel "ein Land, zwei Systeme" gegeben. Seiner Meinung nach sei die Konzeption "ein Land, zwei Systeme" dem "common law marriage" ähnlich. Beide Partner könnten einen Vertrag abschließen, wonach sie gemeinsam "eine 2-Zimmer-Wohnung teilen und jeder für sich seine eigenen Schlüssel haben sollte". Das sei das Wichtigste. 49

Alle diese Modelle wurden von den Wissenschaftlem auf dem Festland als unwissenschaftlich und unannehmbar zurückgewiesen. Denn sie seien der Frage "Souveränität" ausgewichen. Wenn man diese umgehe und von Vereinigung rede, könnte man keineswegs die nationale Einheit erreichen. Und jedes Modell würde der Voraussetzung und der Basis seiner Existenz entbehren.50

47 Vgl. Yang Liyu, s. Anm. 46, und Shen Junshan, s. Anm. 40.

48 Shen Junshan, s. Anm. 40.

49 Ebenda.

50 s. Anm. 17 und 25. 


\section{Souveränität und Regierungsgewalt}

Vor der Unterzeichnung der chinesisch-britischen Gemeinsamen Erklärung wurde eine Auffassung in Hongkong vertreten, daß die VR China die Souveränität über Hongkong zurückerlangen könnte, während die Regierungsgewalt als Mandat weiterhin in den Händen der Briten bleiben sollte.51 Dies war für die chinesische Regierung unannehmbar. Denn aus chinesischer Sicht verbindet sich mit dem Begriff "Souveränität" notwendigerweise die Regierungsgewalt. Die Souverämität und die Regierungsgewalt sind in einem dialektischen Verhältnis untrennbar verbunden. 52 Wie würde das Verhältnis zwischen der Souveränität und der Regierungsgewalt bei der Einführung der Konzeption "ein Land, zwei Systeme" aussehen? Wie würde sich die Staatsgewalt in diesem Falle strukturieren? Nehmen wir auf der Grundlage der chinesisch-britischen Vereinbarung und des Grundgesetzes von Hongkong (Entwurf) Hongkong als Beispiel:

Der Kern der Formel "ein Land, zwei Systeme" ist in den Augen der Festlandchinesen die Hervorhebung von "ein Land", nämlich "ein China". Sie bestehen nach wie vor auf dem "ein China"-Prinzip. "Ein Land" bedeute, daß die Staatssouveränität unteilbar und die chinesische Nation eine Einheit sei.53 In der chinesisch-britischen Gemeinsamen Erklärung heißt es u.a.: "Zur Erhaltung der staatlichen Einheit und territorialen Integrität und aus Erwägungen über die Geschichte Hongkongs und seine Gegebenheiten heraus hat die Volksrepublik China beschlossen, nach der Wiederherstellung der Ausübung der Souveränität über Hongkong in Übereinstimmung von Artikel 31 der Verfassung der VR China ein Sonderverwaltungsgebiet Hongkong einzurichten."54 "Das Sonderverwaltungsgebiet Hongkong wird einen hohen Grad an Autonomie genießen, außer in auswärtigen und Verteidigungsangelegenheiten, die Verantwortlichkeiten der Zentralen Volksregierung bleiben." 55

Daraus geht hervor, daß die VR China nach der Wiedererlangung der Souveränität über Hongkong ein unitaristischer Staat bleiben soll. Die Befugnisse des Sonderverwaltungsgebietes Hongkong sollen von der Zentralen Regierung ableiten. Die Verwaltung des Sondergebietes als eine regionale Regierung solle der höchsten Staatsgewalt und der Zentralen Regierung unterstehen. Die Errichtung des Sonderverwaltungsgebietes soll der Verfassung und den Gesetzen der VR China folgen.56 Konkret heißt das:

51 Zheng Yushuo (Hrsg.), Hongkongs zukünftige Entwicklung, Hongkong 1984, S. 10.

52 s. Anm. 25.

53 Ebenda.

54 s. Anm. 1, BR, Nr. 41, 1984, S. III.

55 Ebenda.

56 s. Anm. 25. 
1. Der Nationale Volkskongreß der VR China soll das Grundgesetz des Sonderverwaltungsgebietes Hongkong erlassen, wonach das kapitalistische System und die Lebensweise in Hongkong innerhalb von 50 Jahren unverändert bleiben können. Das Sonderverwaltungsgebiet Hongkong würde gesetzmäßig mit Exekutiv-, Legislativ- und unabhängigen Judikativbefugnissen einschließlich der der letzten richterlichen Entscheidung sowie gewissen Befugnissen in auswärtigen Angelegenheiten ausgestattet werden. 57

2. Auswärtige Angelegenheiten und die Landesverteidigung sollen ausschließlich von der Zentralen Regierung wahrgenommen werden. Die Zentrale Regierung soll das Recht haben, militärische Kräfte nach Hongkong zu schicken und sie dort zu Verteidigungszwecken zu stationieren. 58

3. Die Zentrale Regierung würde den durch örtliche Wahlen oder Konsultationen gewählten Verwaltungsleiter des Sondergebietes und die von diesem nominierten leitenden Beamten ernennen.59

4. Die Legislative des Sonderverwaltungsgebietes müßte dem Ständigen Ausschuß des Nationalen Volkskongresses die in Übereinstimmung mit dem Grundgesetz und den gesetzlichen Verfahren erlassenen Gesetze zur Archivierung vorlegen.60

5. Emennung und Entlassung der obersten Richter des Sonderverwaltungsgebietes müßte dem Ständigen Ausschuß des Volkskongresses zur Archivierung vorgelegt werden.61

6. Die Verwaltung des Sondergebietes müßte der Zentralen Regierung ihre Haushaltspläne und Budgets zur Archivierung vorlegen.62

7. Die Errichtung ausländischer Wirtschafts- und Handelsmissionen müßte ebenso der Zentralen Regierung vorgelegt werden.63

8. Die Zentrale Regierung könnte die Verwaltung des Sondergebietes auf dem Gebiet der Schiffahrt, des Luftverkehrs sowie bei der Ausgabe von Pässen bevollmächtigen. 64

57 s. 54., vgl ebenso Grundgesetz des Sonderverwaltungsgebietes der VR China Hongkong (Entwurf, weiterhin als GGHK), in: RMRB, 24.02.1989, hier besonders die Einleitung, Art. 5, 19, 149-153.

58 s. Anm. 1, S. XI und ebenso GGHK, Art. 13, 14.

59 s. Anm. 1, S. V und ebenso GGHK, Art. 45.

60 s. Anm. 1, S. VI und ebenso GGHK, Anhang, Art. 5.

61 s. Anm. 1, S. VI und ebenso GGHK, Art. 89.

62 s. Anm. 1, S. II.

63 s. Anm. 1, S. VII und ebenso GGHK, Art. 155.

64 s. Anm. 1, S. VII f. und ebenso GGHK, Art. 124, 132 und 153. 
Andererseits, wie oben erwähnt, würde das Sonderverwaltungsgebiet Hongkong einen hohen Grad an Autonomie genießen. Dadurch würde die VR China bei der Durchführung der Konzeption "ein Land, zwei Systeme" manche Eigenschaften, die normalerweise nur einem Bundesstaat eigen sind, besitzen.65 In mancher Hinsicht sollten die Autonomierechte des Sonderverwaltungsgebietes größer als die der Einzelstaaten innerhalb der 19 Bundesstaaten der heutigen Welt sein.66 Sie finden hauptsächlich folgendermaßen ihren Ausdruck:

1. "Die Verwaltung Hongkongs durch Hongkonger". Die Verwaltung des Sondergebietes Hongkong und dessen Legislative würden aus den dortigen Bewohnem bestehen. Der Verwaltungsleiter sollte durch örtliche Wahlen oder Konsultationen gewählt, ihm sollte die Nominierungsbefugnis der leitenden Beamten der Verwaltung eingeräumt werden. 67

2. Die eigene Gesetzgebungsmacht. Die gesetzgebende Macht Honkongs würde dessen Legislative übertragen, die im Einklang mit dem Grundgesetz und den gesetzlichen Verfahren Gesetze erlassen könnte. Die bisher gültigen Gesetze (nämlich das allgemeine Recht, die Regeln der Billigkeit, die Verordnungen, die untergeordnete Gesetzgebung und das Gewohnheitsrecht) blieben im wesentlichen beibehalten. 68

3. Die Unabhängigkeit der Gerichte. Die Gerichtsbarkeit im Sonderverwaltungsgebiet Hongkong würde dessen Gerichten übertragen, die sie unabhängig und frei von jedem Einfluß ausüben. Das bisher in Hongkong praktizierte Justizsystem würde im wesentlichen beibehalten werden können. Die Befugnis letztendlicher richterlicher Entscheidung des Sonderverwaltungsgebietes würde bei dessen Gerichtshof oberster Instanz liegen.69

4. Die Selbständigkeit des Finanzwesens. Das Sonderverwaltungsgebiet Hongkong könnte sich selbständig mit finanziellen Angelegenheiten, einschließlich der Verteilung seiner Ressourcen und Erstellung seiner Haushaltspläne und Budgets, befassen. Die Zentrale Regierung würde keine Steuer vom Sonderverwaltungsgebiet erheben.70

5. Die Selbständigkeit der Währungspolitik. Die Verwaltung des Sondergebietes könnte ihre Währungs- und Finanzpolitik selbständig festlegen. Der Hongkong-Dollar als die örtliche gesetzmäßige Währung bliebe nach wie vor im Umlauf und frei konvertierbar.

65 Vgl. Yan Jiaqi, s. Anm. 17, S. 191.

66 s. Anm. 24, S. 14.

67 s. Anm. 2, S. V, vgl. ebenso Deng Xiaoping, s. Anm. 9, S. 84.

68 s. Anm. 1, S. VI und ebenso GGHK, Art. 2,8.

69 s. Anm. 1, S. VI f. und ebenso GGHK, Art. 2, 8, 19.

70 s. Anm. 1, S. VII f und ebenso GGHK, Art. 105. 
Die Befugnis für die Ausgabe der Hongkonger Währung würde bei der Regierung des Sonderverwaltungsgebietes liegen. 71

6. Die Beibehaltung der kapitalistischen Wirtschaft. Das Sonderverwaltungsgebiet dürfte die bisher in Hongkong praktizierten kapitalistischen wirtschaftlichen und Handelssysteme beibehalten. Die dortige Regierung könnte selbst ihre Wirtschafts- und Handelspolitik bestimmen. Der Status eines Freihafens würde unverändert bleiben. Hongkong könnte selbständig wirtschaftliche und Handelsbeziehungen mit allen Ländem und Gebieten aufrechterhalten und entwickeln.72

7. Gewisse Befugnisse in auswärtigen Angelegenheiten. Die Regierung des Sonderverwaltungsgebietes Hongkong könnte nach der Bevollmächtigung selber unter dem Namen "Hongkong, China" Beziehungen mit Staaten, Gebieten und einschlägigen internationalen Organisationen in den Bereichen Wirtschaft, Handel, Finanzen und Währung, Schiffahrt, Nachrichtenwesen, Tourismus, Kultur und Sport beibehalten und entwickeln und mit ihnen diesbezügliche Abkommen abschließen und durchführen. Sie könnte sich genauso unter dem Namen "Hongkong, China" an intemationalen Organisationen und Konferenzen, die nicht auf Staaten beschränkt sind, beteiligen u.a.73 Sie würde ermächtigt werden, Pässe des Sonderverwaltungsgebietes Hongkong und andere Reisedokumente auszugeben. 74

8. Das eigene Polizeirecht. Verantwortlich für die Aufrechterhaltung der örtlichen Ordnung würde die Regierung des Sonderverwaltungsgebietes sein. Die von der Zentralen Regierung geschickten militärischen Kräfte dürften sich nicht in die inneren Angelegenheiten Hongkongs einmischen. 75

9. Die Autonomie der Zivilluftfahrt. Das Sonderverwaltungsgebiet würde den Status Hongkongs als ein Zentrum der internationalen und regionalen Luftfahrt aufrechterhalten und könnte selbst für die Routineangelegenheiten und das technische Management der Zivilluftfahrt verantwortlich sein und entsprechende Verpflichtungen erfüllen.76

10. Das eigene Erziehungs- und Bildungssystem. Das bisher in Hongkong praktizierte Erziehungs- und Bildungssystem dürfte beibehalten werden. Lehranstalten aller Art könnten ihre Autonomie genießen.77

71 s. Anm. 1, S. VIII und ebenso GGHK, Art. 109-112.

72 s. Anm. 1, S. VIII und ebenso GGHK, Art. 5, 113.

73 s. Anm. 1, S. X und ebenso GGHK, Art. 115, 149-155.

74 s. Anm. 1, S. XII und ebenso GGHK, Art. 153.

75 s. Anm. 1, S. XI und ebenso GGHK, Art. 14.

76 s. Anm. 1, S. IX und ebenso GGHK, Art. 127-134.

77 s. Anm. 1, S. X und ebenso GGHK, Art. 135-136. 
Obwohl manche Befugnisse der Regierung des Sonderverwaltungsgebietes Hongkong viel größer als die Bundesstaaten und -länder in der heutigen Welt sind, ist der Begriff "Bundesstaat" oder Föderalismus bei der Diskussion über die Formel "ein Land, zwei Systeme" bzw. bei der Auseinandersetzung über die Wiedervereinigung des Landes immer von der chinesischen Führung als unvertretbar zurückgewiesen.78

\section{Ausblick}

Nach Inkrafttreten der chinesisch-britischen Gemeinsamen Erklärung und der chinesischportugiesischen Gemeinsamen Erklärung ist die Konzeption "ein Land, zwei Systeme" verbindlich geworden. $\mathrm{Ob}$ sie durchführbar ist, wird weiterhin lebhaft diskutiert, und es bestehen diametrale Meinungsverschiedenheiten zwischen den Wissenschaftlem auf dem chinesischen Festland einerseits und ihren Kollegen in Hongkong, Taiwan bzw. im Ausland andererseits. 79 Bei den ersteren wird sehr populär die Auffassung vertreten, daß die Konzeption "ein Land, zwei Systeme" praxisbezogen sei und sie sich hinsichtlich der Wiedererlangung der festlandchinesischen Souveränität über Hongkong und Macao als durchführbar und erfolgreich erwiesen habe. 80 Bei den letzteren herrscht jedoch eine große Skepsis gegenüber der praktischen Anwendung der Konzeption. Im Fall Hongkong und Macao konzentriert sich gegenwärtig die Aufmerksamkeit hauptsächlich auf die Frage, ob die gerade auszuarbeitenden Grundgesetze von Hongkong und Macao den beiden Gebieten nach deren Rückgabe 1997 bzw. 1999 die großen Autonomierechte, Stabilität und Prosperität garantieren können.81 Im Fall von Taiwan ist die Skepsis gegenüber der Durchführbarkeit der Formel "ein Land, zwei Systeme" noch viel größer. Denn Hongkong würde nach dem Abzug der Briten machtlos und willenlos gegenüber der VR China sein, und ihm würde nichts übrigbleiben, als die Formel "ein Land mit zwei Systemen" zu akzeptieren. Für Macao würde es auch nicht anders sein. Taiwan hat seine eigenen Streitkräfte, seine politische und rechtliche Struktur. Außerdem besitzt es eine wirtschaftliche Überlegenheit gegenüber dem chinesischen Festland. 82

Xu Jiatun, der Chef der Xinhua-Nachrichtenagentur in Hongkong, bewertet die Konzeption "ein Land, zwei Systeme" als einen "äußerst wichtigen Leitgedanken im Leben unserer Zeit". Sie stelle die Grundlinie der chinesischen Führung zur Verwirklichung der Vereinigung des Landes dar. Diese "schöpferische Entscheidung" eröffne "eine in der Geschichte

78 Weng Songran, s. Anm. 24, und Zhao Ziyang, RMRB, 19.03.1989.

79 s. Anm. 17, 25, 37 und 40.

80 s. Anm. 3 und Anm. 9, S. 91.

81 s. Anm. 37.

82 Qi Xin, Die Lage von Taiwan im Druck durch das Hongkong-Modell, in: The Nineties, Nr. 5 , 1985, und ebenso Gao Yingmao, Die drei Phasen der "Festland-Politik", in: Vereinigungszeitung (Taiwan), 05.08.1988. 
nie dagewesene politische Perspektive" und sie sei eine "große Strategie zum Aufbau des Landes, die im Interesse des Vaterlandes und auf der Grundlage des nationalen Gefühls entwickelt" sei. Nach der Unterzeichnung der chinesisch-britischen Vereinbarung sei diese "wissenschaftliche Konzeption ein politisches Programm und eine Machtstruktur geworden, die in 12 Jahren unbedingt in die Tat umgesetzt und bei der Durchführung zweifellos zum Erfolg geführt" werden müsse. 83

Die Konzeption "ein Land, zwei Systeme" sei nicht nur bei der Regelung der Hongkongbzw. der Taiwan-Frage von großer Bedeutung, sie sei für die Lösung ähnlicher Probleme in der Welt von erheblicher Tragweite. Sie sei in Wirklichkeit eine Produktionsweise und eine entsprechende politisch-rechtliche Ordnung als Hauprumpf in einem Land, wo unter dieser Voraussetzung eine von der Geschichte hinterlassene andere Produktionsweise und dementsprechend ein unterschiedliches politisch-rechtliches System gestattet werden könnte. Gerade wegen der unterschiedlichen Produktionsweisen sei diese schöpferische politische Konzeption zur Überwindung der nationalen Teilung notwendig, die sich nach dem II. Weltkrieg herausgebildet habe. 84

Für die Anwendung der Formel "ein Land, zwei Systeme" auf Taiwan bestehen die Hauptschwierigkeiten darin, daß die Standpunkte der Beijinger bzw. der Taiwaner Behörden zur Wiedervereinigung des Landes diametral entgegengesetzt sind. Die Guomindang-Behörden sind der Ansicht, daß die Wiedervereinigung Chinas zwar notwendig, daß sie aber nur unter "Drei Volksprinzipien" - Nationalismus, Volksrecht und Volkswohlfahrt85 - erreicht werden könne.86 Sie verlangen, daß die Behörden in Beijing auf Gewaltanwendung in der Taiwan, auf die vier Grundprinzipien - Festhalten an dem Sozialismus, Festhalten an der Führung durch die KP Chinas, Festhalten an der volksdemokratischen Diktatur sowie Festhalten an dem Marxismus-Leninismus und den Mao-Zedong-Ideen87 - und schließlich auf die Konzeption "ein Land, zwei Systeme" verzichten müßten.88 Um dem Aufruf der Bejinger Führung von 1979, Handels-, Post- und Schiffahrtsverkehr zwischen den beiden Seiten der Taiwan-Straße wiederherzustellen, wurde eine Dreifaches-Nein-Politik in demselben Jahre in Taibei formuliert - keine offiziellen Kontakte, keine offiziellen Verhandlungen und keine Kompromisse mit der Führung der KP Chinas. 89 Obwohl die Einwohner aus Taiwan

83 s. Anm. 37.

84 s. Anm. 25.

85 Qui Xiao u.a., Lexikon der Politischen Wissenschaft, Chengdu 1986, S. 17.

86 Die wirkliche Lösung der China-Frage - Manifest des XIII. Parteitages der Guomindang, in: Vereinigungszeitung, 14.07.1988.

87 s. Anm. 85, S. 145.

88 Li Dengfei, Vereinigungszeitung, 25.08.1988.

89 Qiu Hongda, ebenda, 17.07.1988. 
außer Beamten und Militärs seit dem November 1987 das Festland besuchen dürfen, wenn sie Familienangehörige dort haben, bleibt diese Nein-Politik nach wie vor bestehen.90

Es wurde der Guomindang-Führung in Taibei vorgeschlagen, diese Dreifaches-Nein-Politik durch eine Politik der "friedlichen Koexistenz", des "friedlichen Wettkampfes" und der "friedlichen Wiedervereinigung" zu ersetzen, vorausgesetzt, daß die Führung in Beijing die obengenannten Forderungen seitens der Behörden in Taibei erfülle.91

Ministerpräsident Yu Guohua wollte nicht nur eine territoriale Vereinigung sehen, sondem es müßte damit "eine politische Demokratisierung, eine wirtschaftliche Liberalisierung, eine gesellschaftliche Pluralisierung und schließlich eine Rückkehr der Kultur an die chinesischen Traditionen" erreicht werden. Auf diese Weise müßten "Freiheit", "Demokratie" und "Gleichberechtigung am Eigentum" verwirklicht werden.92

Bemerkbar ist, daß die Guomindang-Behörden im April 1989 die Formel "ein China, zwei ebenbürtige (paritätische) Regierungen" in Betracht gezogen haben, um damit der Konzeption "ein Land, zwei Systeme" zu begegnen und sich außenpolitisch aus der Isolation herauszuhelfen. Sie wäre ein Kompromiß, entbehrt aber jeder Grundlage im traditionellen Völkerrecht.93

Eine andere Ansicht ist in der Hongkonger Presse zu finden. Die Initiative aus Taibei "ein Land, zwei ebenbürtige Regierungen" könnte sich theoretisch auf vorhandene Beispiele berufen, z.B. Ost- und Westdeutschland, Süd- und Nordkorea. Aber sie würde die Vereinigung Chinas in eine noch weitere Zukunft rücken.94 Ehe sie sich in die Tat umsetzen lasse, müsse sie schon zurückgenommen werden, weil sie ausschließlich als Alternative zur "Festland-Politik" und nicht als konkrete Richtlinie diene.95

Die Formel "ein China, zwei ebenbürtige Regierungen" wäre für die Führung in Beijing faktisch unakzeptabel. Denn in den Augen der Festlandchinesen würde die Teilung verewigen. Außerdem könnte Taiwan der Konzeption "ein Land, zwei Systeme" gemäß nach der eventuellen Wiedervereinigung mit dem Festland nur eine "örtliche Regierung" bilden, die zwar einen hohen Grad an Autonomie genießen, jedoch nicht die Staatssouveränität ausüben dürfe, wie die auswärtigen Angelegenheiten und die Landesverteidigung, es sei

93 Zili Zaobao, 12.04.1989.

94 Xin Bao, 11.04.1989.

95 Zili Wanbao, 17.04.1989. 
denn, daß die Zentralregierung sie mit gewissen Befugnissen auf diesen Gebieten bevollmächtigen wollte.96

Schon von Rechts wegen könnte die Führung in Beijing die obengenannten Forderungen der Guomindang-Behörden nicht erfüllen. Die vier Grundprinzipien sind bereits in der neuen Verfassung der VR China 1982 verbrieft. 97 Nach dieser Ansicht von Deng Xiaoping sollten sie die Basis der chinesischen Politik einschließlich der Hongkong-, der Macao- und der Taiwanpolitik bilden, kurzum, die Konzeption "ein Land, zwei Systeme" könnte auch nur auf der Basis der vier Grundprinzipien formuliert und praktiziert werden. Ohne das sozialistische System auf dem Festland Chinas würde nicht mehr von der Formel "ein Land, zwei Systeme" die Rede sein.98

So wird es ein langer Weg sein, bis sich Bejing und Taibei an den Verhandlungstisch setzen. Und die Formel "ein Land, zwei Systeme" könnte sicherlich nicht so einfach in der Taiwan-Frage ihre Anwendung finden, wie sie sich bei der Wiedererlangung der festlandchinesischen Souveränität über Hongkong und Macao als - einstweilen nur außenpolitisch erfolgreich erwiesen hat. Im Vergleich zu den vier Grundprinzipien dürfte Artikel 31 der Verfassung der VR China99 als Basis für die Regelung der Taiwan-Frage gemäß der Konzeption "ein Land, zwei Systeme" zu schwach sein. Eine hier Abhilfe versprechende Verfassungsreform ist jedoch gegenwärtig in der VR China nicht sichtbar.

96 s. Anm. 53, 54, 55.

97 Einleitung der Verfassung der VR China 1982, s. Anm. 21.

98 Deng Xiaoping, Wichtige Gespräche von Deng Xiaoping (Februar-Juli 1987), Beijing 1987, S. $14 \mathrm{ff}$.

99 s. Anm. 21. 


\section{The Chinese Concept of "One Country, Two Systems"}

\section{By Rongyuan Lin}

The article first discusses, from the point of view of political science, the formative historical background and the process of development of the policy of "one country, two systems", the evolvement of the Chinese leadership's policy towards Taiwan and the application of the notion of "one country, two systems" to the question of Hong Kong and Macao.

The essay emphasises the presentation of China's definition of "one country, two systems", the models for the reunification of China proposed by academic students in China and abroad and the attitudes adopted in this regard by the Chinese government. In addition, the relationship between the central government of a unitary state and a special administrative area enjoying a high degree of autonomy under the model of "one country, two systems" is examined on the basis of the draft "Basic Law for the Special Administrative Area of Hong Kong".

Finally, the author looks ahead at the realistic prospects for application of the formula of "one country, two systems" to the question of Taiwan. He points out that, due to certain wide differences of view and even outright opposition between Peking and Taipeh about the reunification of China, the road to reunification will be very long indeed. 